\title{
Dance therapy improves motor and cognitive functions in patients with Parkinson's disease
}

\author{
Edoardo Rosario de Natale ${ }^{\mathrm{a}}$, Kai Stephan Paulus ${ }^{\mathrm{b}}$, Elena Aiello ${ }^{\mathrm{c}}$, Battistina Sanna ${ }^{\mathrm{c}}$, \\ Andrea Manca $^{\mathrm{a}}$, Giovanni Sotgiu ${ }^{\mathrm{a}}$, Paolo Tranquilli Leali ${ }^{\mathrm{c}}$ and Franca Deriu ${ }^{\mathrm{a}, *}$ \\ ${ }^{a}$ Department of Biomedical Sciences, University of Sassari, Sassari, Italy \\ ${ }^{\mathrm{b}}$ Department of Clinical and Experimental Medicine, University of Sassari, Sassari, Italy \\ ${ }^{\mathrm{c}}$ Department of Surgical and Microsurgical Sciences, University of Sassari, Sassari, Italy
}

\begin{abstract}
.
OBJECTIVE: To explore the effects of Dance Therapy (DT) and Traditional Rehabilitation (TR) on both motor and cognitive domains in Parkinson's Disease patients (PD) with postural instability.

METHODS: Sixteen PD patients with recent history of falls were divided in two groups (Dance Therapy, DT and Traditional Rehabilitation, TR); nine patients received 1-hour DT classes twice per week, completing 20 lessons within 10 weeks; seven patients received a similar cycle of 20 group sessions of 60 minutes TR. Motor (Berg Balance Scale - BBS, Gait Dynamic Index - GDI, Timed Up and Go Test - TUG, 4 Square-Step Test - 4SST, 6-Minute Walking Test - 6MWT) and cognitive measures (Frontal Assessment Battery - FAB, Trail Making Test A \& B - TMT A\&B, Stroop Test) were tested at baseline, after the treatment completion and after 8-week follow-up.

RESULTS: In the DT group, but not in the TR group, motor and cognitive outcomes significantly improved after treatment and retained after follow-up. Significant changes were found for 6MWT $(p=0.028)$, TUG $(p=0.007)$, TMT-A $(p=0.014)$ and TMT-B $(p=0.036)$.

CONCLUSIONS: DT is an unconventional physical therapy for PD patients which effectively impacts on motor (endurance and risk of falls) and non-motor functions (executive functions).
\end{abstract}

Keywords: Rehabilitation, dance therapy, Parkinson's disease, executive functions

\section{Introduction}

In Parkinson's Disease (PD), postural instability, risk of falls and attentional deficits may be driven by alterations of cortical and non-cortical structures (Yarnall et al., 2011). Postural instability constitutes a major risk factor for disability, poor quality of life and long-term hospitalization (Sarter et al., 2014). Dance Therapy (DT) is a promising form of neuro-

*Address for correspondence: Prof. Franca Deriu, Department of Biomedical Sciences, University of Sassari, Viale San Pietro 43/b, 07100 - Sassari, Italy. Tel.: +39 079228294; Fax: +39 079228156; E-mail: deriuf@uniss.it. rehabilitation in PD, combining highly task-specific motor and attentional solicitations. Compared with Traditional Rehabilitation (TR), DT seems more effective in improving balance, strength, and movement strategies (De Dreu et al., 2012); however, few works compared DT and TR effects on cognitive domains (Cruise et al., 2011; McKee \& Hackney, 2013). This study proposed to compare DT and TR effects on motor and cognitive domains.

\section{Methods}

PD patients, diagnosed with Gelb's Criteria (Gelb et al., 1999) responders to L-DOPA and with score 
$>25$ on a preliminary Mini Mental State Evaluation screening were consecutively enrolled. At neurological examination, a Unified Parkinson's Disease Rating Scale (UPDRS) was carried out and a history of at least one fall in a previous three-month period, as well as presence of static-dynamic postural instability not related to other medical factors was verified. Furthermore, static and dynamic balance were measured through the following scales: Berg Balance Scale (BBS), Gait Dynamic Index (GDI), Timed Up-and-Go Test (TUG), 4-Square Step Test (4SST), and 6-minutes Walking Test (6MWT). Neuropsychological assessment of executive functions and attentional skills was performed through Frontal Assessment Battery (FAB), Trail Making Test A \& B (TMT A\&B), and Stroop Test. Examiners were blinded to patient allocation. All assessments were carried out in ON phase and were repeated 2 weeks before intervention (PRE), 2 weeks within the end of intervention (POST), and after 8 weeks (FOLLOW UP). The study was approved by the local ethical committee and conforms to the Helsinki declaration.

\subsection{Intervention}

Interventions were aimed at decreasing fall risk, improving static-dynamic balance and stride length, increasing safety during gait and changing the dynamics of double support. Patients were allocated into two arms (DT and TR). Both groups attended plenary classes of 20 sessions lasting 60 minutes each, twice weekly, for 10 weeks. In the DT class (9 patients) sessions were organized as follows: a preliminary warm-up to music (about 5-10 minutes); next, new Tango steps of increasing difficulties were taught, following protocols used for previous studies on Argentine Tango (Hackney \& Earhart, 2010) finally, 5-10 minutes were dedicated to free partnered dance along the dancehall, according to what learnt during the lesson. In TR (7 patients), static and dynamic balance exercises were performed, along with gait training with frequent, unexpected changes of directions while walking along a lane using acoustic cues and exercises for motor coordination, by alternating motion of limbs.

\subsection{Statistical analysis}

Groups were compared with independent Student $t$-tests for the quantitative variables. Continuous variables were compared using ANOVA for repeated measures during 3 time points, using a Tukey's post-hoc test and Cohen's Effect Size. Statistical significance was set at $<0.05$. Statistical analysis was performed using STATA software 13.0 for Windows.

\section{Results}

Sixteen patients, $(67 \pm 6.9$ years old), were included in the study. Two patients dropped out. Table 1 shows the demographic and clinical characteristics of the patients. No falls were reported in both groups during the study.

At baseline, the two groups were not statistically different for the motor and the cognitive domains. Changes in motor and cognitive outcomes detected from PRE to POST and at FOLLOWUP are described in Table 2: motor outcomes showed a significant PRE to POST improvement on 6 MWT $(+16.7 \%)$ and on TUG $(-20.45 \%)$ in the DT group, significantly retained during the FOLLOWUP $(+1.14 \%$ and $-4.4 \%$, respectively) with a significant difference over time. In the TR arm motor scores did not change significantly over time; moreover, analysis of interaction TIME*GROUP showed important effect for TUG scores $\left(F_{(2,13)}=9.599, p\right.$ value $=0.009$ ). For cognitive outcomes changes were appreciated for TMT-A and TMT-B times; they significantly decreased from PRE to POST $(-46.6 \%$ and $-8.3 \%$, respectively) in the DT group, with keeping during the FOLLOW-UP. Cognitive performances of the TR group did not significantly change after intervention; furthermore, a significant interaction of TIME*GROUP was appreciated for TMT-B performances $\left(F_{(2,13)}=5.263 p\right.$-value $\left.=0.04\right)$ and for Stroop time performances $\left(F_{(2,13)}=7.928 p\right.$ value $=0.016$ ).

\section{Discussion}

In this study DT significantly improved some motor and cognitive tests in PD patients followed-up

Table 1

Demographic and clinical features of PD patients

\begin{tabular}{lccc}
\hline Patient characteristics & Dance therapy & $\begin{array}{c}\text { Traditional } \\
\text { rehabilitation }\end{array}$ & $p$-value \\
\hline Mean (SD) Age, years & $66.0(9.15)$ & $70.0(3.16)$ & $n s$ \\
Males (\%) & $7 / 9(77.8)$ & $4 / 7(57.1)$ & $n s$ \\
Mean (SD) Disease & $6.0(2.07)$ & $6.33(2.25)$ & $n s$ \\
Duration, years & & & \\
Hoehn \& Yahr & $2.5(0.7)$ & $2.6(0.6)$ & $n s$ \\
LEDD & $516.5(209.8)$ & $588.8(138.4)$ & $n s$ \\
\hline
\end{tabular}

LEDD: Levodopa Equivalent Daily Dose. $p$-value calculated with Student $t$-Test and Chi square test when appropriate. SD: Standard Deviation. 
Table 2

Scheme of motor and cognitive scores obtained by DT and TR groups at the three times of the study

\begin{tabular}{|c|c|c|c|c|c|c|c|}
\hline Domain & ITEM & GROUP & PRE & POST & FOLLOW-UP & $p$-value & $\eta^{2}$ \\
\hline \multirow[t]{14}{*}{ Motor } & \multirow[t]{2}{*}{ UPDRS } & DT & $38.37(13.01)$ & $36.5(11.27)$ & $35.37(13.43)$ & 0.546 & 0.063 \\
\hline & & TR & $36.67(22.47)$ & 35 (21.6) & $34(22.78)$ & 0.322 & 0.203 \\
\hline & \multirow{2}{*}{ UPDRS III } & DT & $16.75(5.9)$ & $16.5(4.63)$ & $16.12(7.55)$ & 0.911 & 0.013 \\
\hline & & TR & $16.83(8.77)$ & $14.5(8.45)$ & $14(9.9)$ & 0.446 & 0.149 \\
\hline & \multirow[t]{2}{*}{ BBS } & DT & $49.12(5.66)$ & $51.25(4.13)$ & $51.5(5.07)$ & 0.15 & 0.238 \\
\hline & & TR & $47.5(7.53)$ & $49.5(7.23)$ & $48.5(7)$ & 0.28 & 0.225 \\
\hline & \multirow[t]{2}{*}{ GDI } & DT & $20.12(2.75)$ & $20(2.97)$ & $21(1.51)$ & 0.432 & 0.113 \\
\hline & & TR & $19.83(3.19)$ & $20.17(3.12)$ & $19.67(3.56)$ & 0.538 & 0.117 \\
\hline & \multirow[t]{2}{*}{ TUG (s) } & DT & $13.15(4.30)$ & $10.46(3.25)$ & $10.00(2.35)$ & 0.007 & 0.510 \\
\hline & & TR & $11.26(2.97)$ & $11.24(2.87)$ & $12.57(4.05)$ & 0.05 & 0.653 \\
\hline & \multirow[t]{2}{*}{ 4SST (s) } & DT & $12.06(2.65)$ & $11.94(3.32)$ & $11.35(2.12)$ & 0.696 & 0.03 \\
\hline & & TR & $11.2(3.37)$ & $11.74(5.17)$ & $11.89(2.95)$ & 0.854 & 0.03 \\
\hline & \multirow[t]{2}{*}{ 6MWT (m) } & DT & $319.62(78)$ & $373(11.6)$ & 377.25 (124.94) & 0.028 & 0.4 \\
\hline & & TR & $333.3(102.6)$ & $344.33(119.5)$ & $324.33(89.1)$ & 0.424 & 0.158 \\
\hline \multirow[t]{16}{*}{ Cognitive } & \multirow[t]{2}{*}{ FAB } & DT & $13.62(1.92)$ & $15(3.56)$ & $15.25(1.98)$ & 0.119 & 0.262 \\
\hline & & TR & $11.83(3.49)$ & $12.5(3.62)$ & $12.67(3.08)$ & 0.382 & 0.175 \\
\hline & \multirow[t]{2}{*}{ STROOP III (time) } & DT & $75.75(34.79)$ & $63.87(54.22)$ & $42.87(19.35)$ & 0.05 & 0.34 \\
\hline & & TR & $52.33(11.94)$ & $56(15.4)$ & $39.5(14.54)$ & 0.613 & 0.093 \\
\hline & \multirow[t]{2}{*}{ STROOP interf (time) } & DT & $49.44(31.15)$ & $43.94(47.08)$ & $24.69(16.27)$ & 0.09 & 0.286 \\
\hline & & TR & $30.25(10.85)$ & $32.42(14.03)$ & $26.25(13.23)$ & 0.638 & 0.086 \\
\hline & \multirow[t]{2}{*}{ STROOP III (errors) } & DT & $4.5(2.98)$ & $3.25(4.65)$ & $3.12(4.45)$ & 0.355 & 0.128 \\
\hline & & TR & $5.33(5)$ & $3.67(4.13)$ & $4.67(3.83)$ & 0.564 & 0.108 \\
\hline & \multirow[t]{2}{*}{ STROOP interf. (errors) } & DT & $4(2.81)$ & $3.19(4.69)$ & $2.87(4.41)$ & 0.543 & 0.083 \\
\hline & & TR & $4.25(4.85)$ & $2.75(3.3)$ & $4(3.48)$ & 0.586 & 0.101 \\
\hline & \multirow[t]{2}{*}{ TMT-A (s) } & DT & $63.62(56.1)$ & $34(28.97)$ & $31.37(20.98)$ & 0.014 & 0.456 \\
\hline & & TR & $76.83(85.09)$ & $110(100.79)$ & $69.67(60.58)$ & 0.166 & 0.302 \\
\hline & \multirow[t]{2}{*}{ TMT-B (s) } & DT & $142.5(96.29)$ & $116.37(100.34)$ & $76.12(90.04)$ & 0.036 & 0.379 \\
\hline & & TR & $103.16(105.74)$ & $127.17(107.79)$ & $116.33(106.35)$ & 0.415 & 0.137 \\
\hline & \multirow[t]{2}{*}{ TMT B-A } & DT & $79.12(85.93)$ & $82.62(83.2)$ & $36(72.22)$ & 0.284 & 0.164 \\
\hline & & TR & $26.67(77.41)$ & $17.5(82.63)$ & $47(66.13)$ & 0.626 & 0.089 \\
\hline
\end{tabular}

All values are expressed as mean (Standard Deviation) DT: Dance Therapy TR: Traditional Rehabilitation UPDRS: Unified Parkinson's Disease Rating Scale; BBS: Berg Balance Scale; GDI: Gait Dynamic Index; TUG: Timed Up-and-Go; 4SST: 4 Squared-Step Test; 6MWT: 6-Minutes Walking Test; FAB: Frontal Assessment Battery; TMT: Trail Making Test. ANOVA for repeated measures with effect size $\left(\eta^{2}\right)$. Bold: $p<0.05$.

in an 8-week period. By contrast, TR group showed non-consistent and non-continuous modifications.

The degree of motor changes in the DT group as well as improvements in cognitive outcomes, particularly executive functions confirm findings reported elsewhere (McKee \& Hackney, 2013; Hackney \& Earhart, 2010).

There is a bulk of emerging literature about the potential beneficial effect of physical exercise on prevention (Ahlskog, 2011) and limitation of motor symptoms (Dibble et al., 2009) in PD. DT constitutes a highly-tasked exercise for PD patients, simulating balance perturbations encountered in daily life and encompassing high-demanding pre-determined movements, cognitive strategies and sudden changes in direction. DT stresses the dual-tasking movements, preparing patients to cope with an important cause of falls. This beneficial effect is further confirmed by our results. Additionally, physical exercise in PD also affects cognitive domains, ameliorating executive functions (Cruise et al., 2011). In our study, the moderate but statistically significant improvements on cognitive tests following DT were retained after follow-up, in line with previous reports for Brooks Spatial Test (McKee \& Hackney, 2013). This suggests that DT might influence higher cortical functions for a long period. Attentional deficits and risk of falls are strictly associated with an incapability of the patient to focus on motor/postural and cognitive tasks when they are combined (Yarnall et al., 2011; Sarter et al., 2014). DT, for its intrinsic characteristics, might train the subject to properly address such tasks.

Motor and cognitive results assessed in the DT group were not detected in the TR cohort. Although TR was performed in group classes like DT to avoid potential effects of social interaction on outcomes, one of the most relevant characteristics of DT is its entertaining nature and facilitation of participants' involvement (Hackney \& Earhart, 2010). This might 
partially explain the superior DT effect over time. It is plausible that TR duration, adapted to that of the DT program, was too short in order to allow the achievement of significant results.

In conclusion, DT is an entertaining unconventional physical therapy for PD patients and may have relevant impacts on motor and non-motor functions.

\section{Acknowledgments}

The Authors would like to thank the dance instructor Mr. Bruno Pitzolu for his professional contribution to the dance therapy; Prof. Virgilio Agnetti for clinical supervision to the study; Dr. Francesca Ginatempo and Giovanna Pilurzi for their assistance to patients during dance sessions and Dr Caterina Casa for her professional assistance to patients during the traditional rehabilitation sessions. The work was funded by Fondazione Banco di Sardegna (FBS) Grant 2012.0745.

\section{Conflict of interest}

All authors declare no conflicts of interest.

\section{References}

Ahlskog, J. E. (2011). Does vigorous exercise have a neuroprotective effect in Parkinson disease? Neurology, 77, 288-294

Cruise, K. E., Bucks, R. S., Poftus, A. M., Newton, R. U., Pegoraro R., \& Thomas M. G. (2011). Exercise and Parkinson's: Benefits and quality of life. Acta Neurol Scand, 123, 13-19.

De Dreu, M. J., Van der Wilk, A. S., Poppe, E., Kwakkel, G., \& van Wegen E. E. (2012). Rehabilitation, exercise therapy and music in patients with Parkinson's disease: A meta-analysis of the effects of music-based movement therapy on walking ability, balance and quality of life, Parkinsonism Relat Disord, (Suppl.1), S114-S119

Dibble, L. E., Addison, O., \& Papa, E. (2009). The effects of exercise in persons with Parkinson's disease: A systematic review across the disability spectrum. J Neurol Phys Ther, 33, 14-26.

Gelb, D. J., Oliver, E., \& Gilman, S. (1999). Diagnostic criteria for Parkinson's disease. Arch Neurol, 56, 33-39.

Hackney, M. E., \& Earhart, G. M. (2010). Effects of dance on gait and balance in Parkinson's disease: A comparison of partnered and nonpartnered dance movement. Neurorehabil Neural Repair, 24, 384-392.

McKee, K. E., \& Hackney, M. E. (2013). The effects of adapted tango on spatial cognition and disease severity in Parkinson's disease. J Mot Behav, 45, 519-529.

Sarter, M., Albin, R. L., Cucinski, A., \& Lustig, C. (2014). Where attention falls: Increased risk of falls from the converging impact of cortical cholinergic and midbrain dopamine loss on striatal function. Exp Neurol, 257, 120-129.

Yarnall, A., Rochester, L., \& Burn, D. J. (2011). The interplay of cholinergic function, attention, and falls in Parkinson's disease. Mov Disord, 26, 2496-2503. 